\title{
Revascularizing coronary artery disease in patients undergoing transcatheter aortic valve implantation
}

\author{
Rafail A. Kotronias ${ }^{1,2}$, Mamas A. Mamas ${ }^{1,3}$, Rodrigo Bagur ${ }^{1,4,5}$ \\ ${ }^{1}$ Keele Cardiovascular Research Group, Institute for Applied Clinical Science and Centre for Prognosis Research, Institute of Primary Care and \\ Health Sciences, University of Keele, Stoke-on-Trent, UK; ${ }^{2}$ Oxford University Clinical Academic Graduate School, Oxford University, Oxford, UK; \\ ${ }^{3}$ The Heart Centre, Royal Stoke Hospital, University Hospital of North Midlands Trust, Stoke-on-Trent, UK; ${ }^{4}$ Division of Cardiology, Department \\ of Medicine, London Health Sciences Centre, London, Ontario, Canada; ${ }^{5}$ Department of Epidemiology and Biostatistics, Schulich School of \\ Medicine \& Dentistry, Western University, London, Ontario, Canada \\ Correspondence to: Rodrigo Bagur, MD, PhD, FAHA. University Hospital, London Health Sciences Centre, 339 Windermere Road, London, Ontario \\ N6A 5A5, Canada. Email: rodrigobagur@yahoo.com. \\ Provenance: This is an invited article commissioned by Section Editor Dr. Hui-Ping Zhang (Section Editor, Department of Cardiology, Beijing \\ Hospital, the Fifth Affiliated Hospital of Peking University, Beijing, China). \\ Response to: Piccolo R, Franzone A, Pilgrim T. Coronary artery disease and myocardial revascularization in patients undergoing transcatheter aortic \\ valve replacement. J Thorac Dis 2017;9:4219-21.
}

Submitted Nov 14, 2017. Accepted for publication Nov 21, 2017.

doi: $10.21037 /$ jtd.2017.11.138

View this article at: http://dx.doi.org/10.21037/jtd.2017.11.138

Coronary artery disease (CAD) is common among patients undergoing transcatheter aortic valve implantation (TAVI) with a prevalence of up to $75 \%$, and no clear recommendations around its treatment (1) and conflicting evidence around whether to perform coronary revascularization or not. Moreover, the role of revascularization on long-term morbidity and mortality is still not clear in octogenarians (2). Piccolo and colleagues (3) have provided an interesting editorial comment on our work (1), and further pointed out controversies with regards to revascularization of patients with $\mathrm{CAD}$ and undergoing TAVI.

The authors also commented on their own results (4), based on an elegant age- and gender-matched analysis where they found a significant increase in the composite of cardiovascular death, myocardial infarction, or stroke at 1-year among TAVI patients with CAD. However, it should be highlighted that the authors found a similar risk of ischemic events during TAVI procedures among patients without CAD as compared to the matched population with CAD. Even when the complexity and severity of CAD is considered and the SYNTAX score is used to stratify CAD severity, the evidence is mixed with regards to mortality outcomes $(5,6)$. Moreover, emerging data on completeness of revascularization is also conflicting. Indeed, while Van Mieghem and colleagues (7) suggested no influence of completeness of revascularization on mortality, a recent large analysis showed that incompleteness of revascularization and more severe $\mathrm{CAD}$ were independent predictors of mortality (6).

Our findings indicate no benefit in 30-day and 1-year outcomes with a revascularization strategy. Notably, revascularized patients were at higher-risk of major vascular complications, although data were derived from percutaneous coronary intervention (PCI) undertaken through the transfemoral approach and much of the data analysed was subject to the inherent limitations of observational registries such as selection biases and unmeasured confounding.

\section{Revascularization strategies}

In terms of symptoms assessment, it is often difficult to rely upon them in this population and tools conventionally used in patients with stable angina to guide revascularization (i.e., fractional flow reserve) are still not well validated and widely used in the setting of severe aortic stenosis. Hence, it is reasonable to percutaneously revascularize ostial or 
proximal lesions located in major epicardial vessels that supply significant areas of myocardium, particularly given that this may be technically more challenging post TAVI. Importantly, should the decision for revascularization be taken, we strongly recommend the use of radial artery approach for PCI as the default access in patients undergoing TAVI, whether a priori or concomitant. Indeed, the use of the radial access site (compared to femoral) in PCI is associated with a significant reduction in major vascular and bleeding complications (8-10) particularly when the transfemoral approach is the preferred access site for TAVI (11).

\section{Timing of revascularization}

The temporal relation of revascularizing coronary lesions to TAVI has not been extensively studied, though a concomitant approach might be seen more attractive when considering the more "controlled" environment during TAVI. The same-setting or concomitant approach is also favoured for ostial lesions with high-risk features for coronary obstruction, as coronary protection strategies with a guidewire can be adopted (12). This strategy might also be beneficial as it minimises pre-procedural dualantiplatelet therapy exposure and thus bleeding risks. In terms of patients with chronic kidney disease, until more data is available, an a priori approach would be advocated to minimize contrast dye and reduce the risk of acute kidney injury, a known independent predictor of mortality in TAVI patients $(13,14)$.

Importantly, if revascularization is not a priori or concomitantly performed and thus, a deferral strategy is chosen, even though post-TAVI PCI is rarely needed and data suggest is feasible in this setting, it can sometimes be challenging (15-19). Hence, performing TAVI with a device that does not jail the coronary ostia would, perhaps, be advisable $(15-17,19)$. In addition, while TAVI is nowadays extended to lower-risk, younger, and less morbid patients, also exhibiting a longer life-expectancy (20), it may be reasonable to proceed with coronary revascularization to prevent the aforementioned potential issue of coronary arteries accessibility should CAD progresses in the future.

Even though physicians are eagerly awaiting the results of the ACTIVATION study (21), one should bear in mind that this is a non-inferiority design trial. Hence, in the absence of solid randomized data showing superiority results that can further guide clinical decision making, individualized risk-benefit assessments should be undertaken based on patient-risk profile, symptoms and life-expectancy. This pragmatic patient-centered approach has been proposed to guide eligibility of choosing TAVI versus surgical aortic valve replacement in the form of the valve durability to life expectancy ratio (20). Certainly, this concept could also assist decision making in the setting of revascularizing $\mathrm{CAD}$ in TAVI candidates by incorporating the benefit and harm of PCI, also bearing in mind the predicted life-expectancy in this subset of patients. With the rapid expansion of the field to include younger patients, a concerted back to basics approach to study the significance of CAD in patients with severe aortic stenosis is warranted. Whilst elective revascularization of CAD in patients with stable angina has not been shown to improve hard clinical outcomes in trials such as the COURAGE (22), there is no high-quality data in patients with severe aortic stenosis. Given the conflicting nature of data available outlined above, we propose a framework that aims to ensure safety in real world clinical practice (Figure 1).

\section{Conclusions}

The decision of revascularizing CAD in the context of TAVI still relies heavily upon the TAVI heart team's clinical judgment along with patient's preferences and values.

\section{Acknowledgements}

None.

\section{Footnote}

Conflicts of Interest: The authors have no conflicts of interest to declare. 


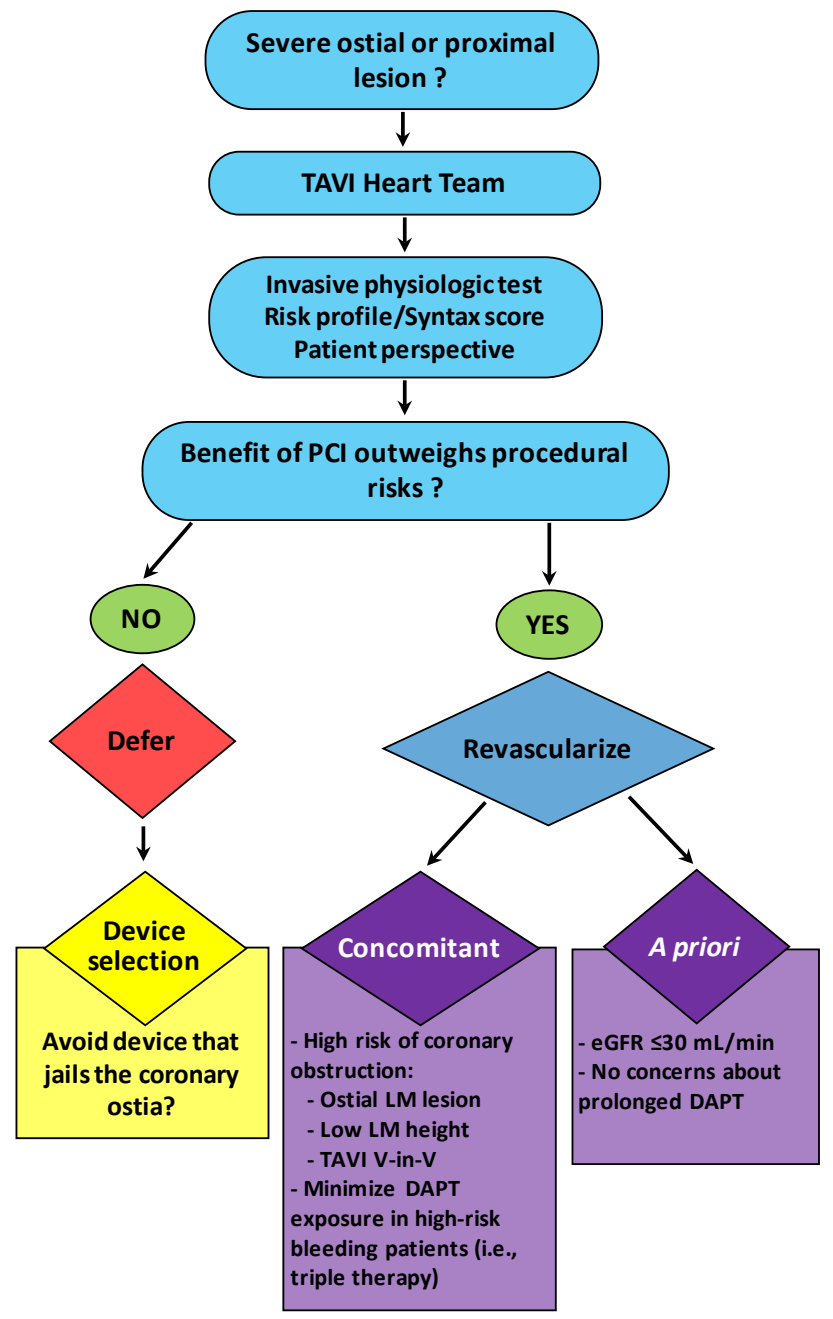

Figure 1 Pragmatic patient-centered approach to guide decision making in the setting of candidates presenting with coronary artery disease and undergoing transcatheter aortic valve implantation (TAVI). PCI, percutaneous coronary intervention; LM, left main; $\mathrm{V}$-in-V, valve in valve; DAPT, dual-antiplatelet therapy; eGFR, estimated glomerular filtration rate. Triple therapy refers to DAPT plus an oral anticoagulant.

\section{References}

1. Kotronias RA, Kwok CS, George S, et al. Transcatheter Aortic Valve Implantation With or Without Percutaneous Coronary Artery Revascularization Strategy: A Systematic Review and Meta-Analysis. J Am Heart Assoc 2017;6. pii: e005960.

2. Dell'Amore A, Aquino TM, Pagliaro M, et al. Aortic valve replacement with and without combined coronary bypass grafts in very elderly patients: early and long-term results.
Eur J Cardiothorac Surg 2012;41:491-8.

3. Piccolo R, Franzone A, Pilgrim T. Coronary artery disease and myocardial revascularization in patients undergoing transcatheter aortic valve replacement. J Thorac Dis 2017;9:4219-21.

4. Franzone A, Stortecky S, Räber L, et al. Effects of coronary artery disease in patients undergoing transcatheter aortic valve implantation: A study of age- and gender-matched cohorts. Int J Cardiol 2017;243:150-5.

5. Stefanini GG, Stortecky S, Cao D, et al. Coronary artery disease severity and aortic stenosis: clinical outcomes according to SYNTAX score in patients undergoing transcatheter aortic valve implantation. Eur Heart J 2014;35:2530-40.

6. Witberg G, Regev E, Chen S, et al. The Prognostic Effects of Coronary Disease Severity and Completeness of Revascularization on Mortality in Patients Undergoing Transcatheter Aortic Valve Replacement. JACC Cardiovasc Interv 2017;10:1428-35.

7. Van Mieghem NM, van der Boon RM, Faqiri E, et al. Complete revascularization is not a prerequisite for success in current transcatheter aortic valve implantation practice. JACC Cardiovasc Interv 2013;6:867-75.

8. Bagur R, Bertrand OF, Rodes-Cabau J, et al. Comparison of outcomes in patients $>$ or $=70$ years versus $<70$ years after transradial coronary stenting with maximal antiplatelet therapy for acute coronary syndrome. Am J Cardiol 2009;104:624-9.

9. Ratib K, Mamas MA, Anderson SG, et al. Access Site Practice and Procedural Outcomes in Relation to Clinical Presentation in 439,947 Patients Undergoing Percutaneous Coronary Intervention in the United Kingdom. JACC Cardiovasc Interv 2015;8:20-9.

10. Ferrante G, Rao SV, Jüni P, et al. Radial Versus Femoral Access for Coronary Interventions Across the Entire Spectrum of Patients With Coronary Artery Disease: A Meta-Analysis of Randomized Trials. JACC Cardiovasc Interv 2016;9:1419-34.

11. Vandvik PO, Otto CM, Siemieniuk RA, et al. Transcatheter or surgical aortic valve replacement for patients with severe, symptomatic, aortic stenosis at low to intermediate surgical risk: a clinical practice guideline. BMJ 2016;354:i5085.

12. Chakravarty T, Sharma R, Abramowitz Y, et al. Outcomes in Patients With Transcatheter Aortic Valve Replacement and Left Main Stenting: The TAVR-LM Registry. J Am Coll Cardiol 2016;67:951-60.

13. Bagur R, Webb JG, Nietlispach F, et al. Acute kidney 
injury following transcatheter aortic valve implantation: predictive factors, prognostic value, and comparison with surgical aortic valve replacement. Eur Heart J 2010;31:865-74.

14. Bagur R, Rodes-Cabau J, Doyle D, et al. Usefulness of TEE as the primary imaging technique to guide transcatheter transapical aortic valve implantation. JACC Cardiovasc Imaging 2011;4:115-24.

15. Bagur R, Dumont E, Doyle D, et al. Coronary ostia stenosis after transcatheter aortic valve implantation. JACC Cardiovasc Interv 2010;3:253-5.

16. Blumenstein J, Kim WK, Liebetrau C, et al. Challenges of coronary angiography and intervention in patients previously treated by TAVI. Clin Res Cardiol 2015;104:632-9.

17. Allali A, El-Mawardy M, Schwarz B, et al. Incidence, feasibility and outcome of percutaneous coronary intervention after transcatheter aortic valve implantation with a self-expanding prosthesis. Results from a single

Cite this article as: Kotronias RA, Mamas MA, Bagur R. Revascularizing coronary artery disease in patients undergoing transcatheter aortic valve implantation. J Thorac Dis 2018;10(1):E79-E82. doi: 10.21037/jtd.2017.11.138 center experience. Cardiovasc Revasc Med 2016;17:391-8.

18. Jackson M, Williams PD. Coronary access following TAVI - Selective coronary engagement using balloon-assisted tracking of a guide catheter extension. Cardiovasc Revasc Med 2017. [Epub ahead of print].

19. Zivelonghi C, Pesarini G, Scarsini R, et al. Coronary Catheterization and Percutaneous Interventions After Transcatheter Aortic Valve Implantation. Am J Cardiol 2017;120:625-31.

20. Bagur R, Pibarot P, Otto CM. Importance of the valve durability-life expectancy ratio in selection of a prosthetic aortic valve. Heart 2017;103:1756-9.

21. Khawaja MZ, Wang D, Pocock S, et al. The percutaneous coronary intervention prior to transcatheter aortic valve implantation (ACTIVATION) trial: study protocol for a randomized controlled trial. Trials 2014;15:300.

22. Sedlis SP, Hartigan PM, Teo KK, et al. Effect of PCI on Long-Term Survival in Patients with Stable Ischemic Heart Disease. N Engl J Med 2015;373:1937-46. 